\title{
FORMATION OF KINEMATIC SUBSYSTEMS IN STELLAR SPIRAL-SPIRAL MERGERS
}

\author{
Marc Balcells and Antonio César González \\ Instituto de Astrofísica de Canarias, 38200 La Laguna, Tenerife, Spain \\ Received 1998 June 9; accepted 1998 July 28; published 1998 August 27
}

\begin{abstract}
We show that kinematically peculiar cores may be generated in stellar spiral-spiral mergers. Kinematic segregation appears as the central bulges transport orbital angular momentum inward to the center of the remnant, while the outer parts keep the spin signature of the precursor disks. The peculiar core is composed mostly of bulge material, and its size best matches that of observed peculiar cores for mergers with unequal galaxy masses $(\sim 2: 1)$. Population decoupling is predicted by the rapid radial decrease of the bulge fraction in the remnant. In this scenario, a starburst may pump up the remnant metallicity, but otherwise the high metallicity of the kinematically decoupled core is built on the high metal content of the inner bulges rather than on a high selfenrichment of a population built from scratch out of the precursors' gas. Mergers with galaxy mass ratios 3:1 and above generate disk galaxies with counterrotating bulges.
\end{abstract}

Subject headings: galaxies: abundances — galaxies: elliptical and lenticular, $\mathrm{cD}$ - galaxies: interactions galaxies: kinematics and dynamics - galaxies: peculiar

\section{INTRODUCTION}

Kinematically decoupled cores (KDCs) are likely to form by mergers, since they are unlikely outcomes of a monolythic collapse. The large- and small-elliptical (eE) merger model (Balcells \& Quinn 1990, hereafter BQ90) predicts most of the dynamical properties of KDCs, but it does not account for the inward reddening of elliptical cores. If anything, nuclei turn out slightly bluer because secondary material, which is bluer as a result of the color-magnitude relation of elliptical galaxies, dominates the surface brightness at small radii.

Population signatures in some KDCs suggest what has been termed "population decoupling," typically a change in the inward slope of a population index such as $\mathrm{Mg}_{2}$ (Davies, Sadlier, \& Peletier 1993; Hau, Carter, \& Balcells 1998, hereafter HCB). A starburst during the process that creates the KDC might account for population decoupling (Surma \& Bender 1995; HCB), although a truncated star formation history sometimes explains the observations (HCB). The former process suggests a merger of metal-rich galaxies. Hernquist \& Barnes (1991, hereafter HB91) present a gravity plus hydrodynamics merger simulation that results in a nuclear counterrotating gas disk. A stellar counterrotating core would presumably result if a starburst turns the gas disk into stars.

In the gasdynamical formation mechanism, the kinematically peculiar component is built entirely in the aftermath of a single merger. We have investigated merger schemes in which the KDC population has stronger links to the stellar populations of the parent galaxies. Here we describe a mechanism for angular momentum segregation in spiral-spiral (S-S) mergers, which can produce KDCs by stellar-dynamical processes alone. The underlying physics is similar to that of the BQ90 eE mergers: the bulges deposit the orbital angular momentum in the center after sinking via dynamical friction, while the outer parts rotate according to the initial orientation of the precursors' spin. If both disks rotate nearly opposite to the orbit, a counterrotating core results.

\section{MERGER MODEL DETAILS}

We used Kuijken-Dubinski (Kuijken \& Dubinski 1995) diskbulge-halo (dbh) models as initial conditions. Four types of galaxy models were used in the merger simulations. Model parameters are given in Table 1, which lists total mass $M_{\text {tor }}$, dark-to-luminous and bulge-to-disk mass ratios, bulge halfmass ratio, disk scale length, disk maximum extent, bulge central density, and the number of particles for halo, disk, and bulge. The first set has dbh masses matching the "minimum halo" Milky Way model of Kuijken \& Dubinski (1995), scaled to total mass unity (model A in Table 1). For experiments involving unequal-mass galaxies, the A model was scaled homologously (models B and C in Table 1). In model D, the total mass was set to 1 and the bulge-to-disk ratio was decreased. Halos were spherical, and both bulges and halos had no net rotation. Physical units that match model A to the Milky Way are $M=3.24 \times 10^{11} M_{\odot}, R=14.0 \mathrm{kpc}$, and $V=315 \mathrm{~km} \mathrm{~s}^{-1}$.

In all of the merger simulations, two dbh galaxies are placed on a nearly parabolic, interpenetrating orbit in the $(x, y)$-plane, with both spins at more than $90^{\circ}$ from the orbital angular momentum. Detailed orbital parameters for eight merger models are given in Table 2. Galaxies start on the $x$-axis. $\theta_{i}$ and $\phi_{i}$ describe the disk spin orientation in spherical coordinates. These experiments allow us to perform basic tests on how core parameters depend on the orbital angular momentum $\boldsymbol{J}_{\text {orb }}$, spin inclination, bulge mass, and galaxy mass ratio.

Merger simulations were run with Hernquist's version of the tree code (Hernquist 1990) on a SGI Power Challenge. Softening was always set at one-fifth of the bulge half-mass radius. The tolerance parameter was 0.8 , and quadrupole terms were included in the force calculation. The number of particles in the experiments is admittedly low but sufficient for the types of observables we are after. Runs with the model galaxy in isolation showed that bulge, disk, and halo are stable for the duration of the merger models; disk thickening is very low, and the bulge density profile is perfectly maintained, showing that the more massive halo particles do not artificially heat the system over the relevant timescales.

\section{THE ONSET OF NUCLEAR COUNTERROTATION}

In all of the experiments, the two model galaxies readily merge owing to the braking effect of the halos and to the fact that the orbit is subparabolic. Because the disks spin in a retrograde direction to the orbit, spin-orbit coupling is weak, and 
TABLE 1

Galaxy Model Parameters

\begin{tabular}{lcccccccccc}
\hline \hline Model & $M_{\text {tot }}$ & $M_{H} / M_{L}$ & $M_{B} / M_{D}$ & $R_{1 / 2, B}$ & $h_{D}$ & $R_{\max , D}$ & $\rho_{0, B}$ & $N_{H}$ & $N_{D}$ & $N_{B}$ \\
\hline $\mathrm{A} \ldots \ldots$ & 1 & 4.15 & 0.5 & 0.063 & 0.322 & 1.61 & 108.40 & 10000 & 9000 & 6000 \\
$\mathrm{~B} \ldots \ldots$. & 2 & 4.15 & 0.5 & 0.089 & 0.455 & 2.28 & 58.17 & 10000 & 9000 & 6000 \\
$\mathrm{C} \ldots \ldots$ & 3 & 4.15 & 0.5 & 0.108 & 0.558 & 2.79 & 63.45 & 10000 & 9000 & 6000 \\
$\mathrm{D} \ldots \ldots$ & 1 & 6.41 & 0.2 & 0.073 & 0.376 & 1.88 & 27.69 & 15000 & 9000 & 3000 \\
\hline
\end{tabular}

TABLE 2

Merger Simulation Parameters

\begin{tabular}{ccccccccccc}
\hline \hline Run & Models & $M_{2} / M_{1}$ & $M_{B} / M_{D}$ & $E_{\text {orb }}$ & $\left|J_{\text {orb }}\right|$ & $r_{\text {peri }}$ & $\theta_{1}$ & $\phi_{1}$ & $\theta_{2}$ & $\phi_{2}$ \\
\hline $1 \ldots \ldots$ & A + A & 1 & 0.5 & -0.562 & 1.19 & 1.80 & 135 & 45 & 135 & -135 \\
$2 \ldots \ldots$ & A + A & 1 & 0.5 & -0.562 & 1.18 & 1.80 & 150 & 45 & 150 & -135 \\
$3 \ldots \ldots$ & A + A & 1 & 0.5 & -0.645 & 0.82 & 0.90 & 135 & 45 & 135 & -135 \\
$4 \ldots \ldots$ & A + A & 1 & 0.5 & -0.622 & 0.88 & 1.05 & 150 & 45 & 150 & -135 \\
$5 \ldots \ldots$ & A + B & 2 & 0.5 & -1.084 & 1.91 & 0.90 & 135 & 45 & 135 & -135 \\
$6 \ldots \ldots$ & A + C & 3 & 0.5 & -1.977 & 1.67 & 0.90 & 135 & 45 & 135 & -135 \\
$7 \ldots \ldots$ & D + D & 1 & 0.2 & -0.512 & 1.24 & 1.80 & 135 & 45 & 135 & -135 \\
$8 \ldots \ldots$ & D + D & 1 & 0.2 & -0.569 & 0.95 & 1.05 & 135 & 45 & 135 & -135 \\
\hline
\end{tabular}

therefore tidal tails do not appear. Global parameters of the luminous matter of the remnants are given in Table 3, which shows the half-mass ratio, the central velocity dispersion, the ratio $V_{\max } / \sigma_{0}$, the counterrotation velocity and radius, and the figure ellipticity for the viewing angle chosen to plot rotation curves (see below). In the equal-mass cases, the remnant rotates slowly. Because of the retrograde nature of the merger, ellipticity does not scale with the rotation parameter $V / \sigma_{0}$. Rather, the remnant ellipticity is higher when the disk spins start out more closely antiparallel to $\boldsymbol{J}_{\text {orb }}$. In the unequal-mass cases, rotation and flattening are progressively more pronounced as the mass ratio increases.

Figure 1 shows major axis rotation curves for model merger 3 . We use this system as a canonical merger to illustrate the dynamical processes that lead to kinematic substructure. In this and other rotation curves shown, material from the bulges is depicted with crosses, material from the disks is depicted with stars, material from galaxy 1 (bulge plus disk) is depicted with squares, material from galaxy 2 (bulge plus disk) is depicted with triangles, and total luminous rotation curves are depicted with circles. The system is viewed along a direction perpendicular to both angular momenta of the luminous material originally belonging to the primary and the secondary galaxy, and the slit is placed in the projected major axis of the resultant figure. The top panel shows that the central region rotates in the opposite sense to the outer parts. The rotation curves of material originally belonging to galaxy 1 and 2 are almost identical owing to the symmetry properties of the merger.

The velocity profiles of the particles coming from the disk and bulge of each galaxy (Fig. 1, middle and bottom) show

TABLE 3

Parameters of Merger Remnants

\begin{tabular}{ccccccc}
\hline \hline Run & $R_{1 / 2}$ & $\sigma_{0}$ & $V_{\max } / \sigma_{0}$ & $V_{\text {cr }}$ & $R_{\text {cr }}$ & $\epsilon$ \\
\hline $1 \ldots \ldots$ & 0.32 & 0.61 & 0.17 & 0.23 & 0.53 & 0.175 \\
$2 \ldots \ldots$ & 0.33 & 0.63 & 0.15 & 0.19 & 0.41 & 0.439 \\
$3 \ldots \ldots$ & 0.31 & 0.60 & 0.22 & 0.26 & 0.47 & 0.187 \\
$4 \ldots \ldots$ & 0.32 & 0.60 & 0.30 & 0.23 & 0.48 & 0.360 \\
$5 \ldots \ldots$ & 0.41 & 0.66 & 0.39 & 0.13 & 0.29 & 0.357 \\
$6 \ldots \ldots$ & 0.46 & 0.68 & 0.64 & 0.07 & 0.14 & 0.420 \\
$7 \ldots \ldots$ & 0.64 & 0.50 & 0.25 & 0.06 & 0.80 & 0.363 \\
$8 \ldots \ldots$ & 0.62 & 0.43 & 0.34 & 0.12 & 0.84 & 0.133 \\
\hline
\end{tabular}

that the bulge particles counterrotate and give the counterrotating signature to the total rotation curve. Bulge and disk materials spin opposite to each other in the remnant.

The alignment of the final rotation of the bulge material with the initial $\boldsymbol{J}_{\text {orb }}$ stems from a well-known law of dissipationless merging: the most bound objects gain binding energy, and the less bound objects lose it. Bulges sink by dynamical friction and deposit in the remnant core any $\boldsymbol{J}_{\text {orb }}$ they have not trans-

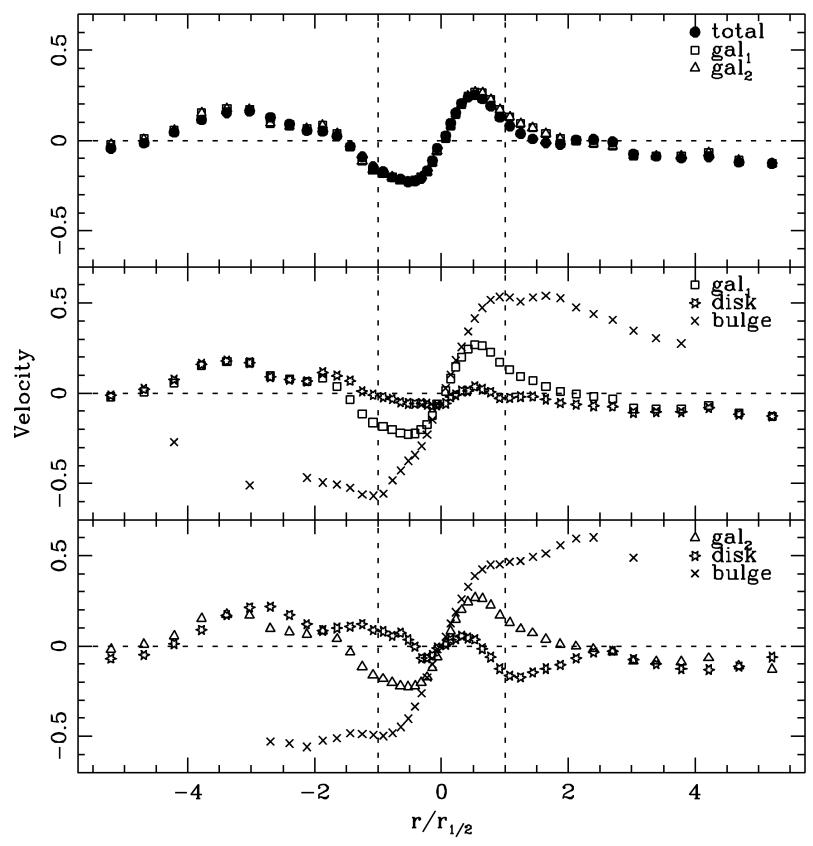

FIG. 1.-Top: Major axis rotation curve for a merger of equal-mass dbh galaxies with $M_{B} / M_{D}=0.5$. Circles: total rotation curve of luminous matter. Squares: rotation curve for matter originally belonging to galaxy 1 . Triangles: rotation curve for matter originally belonging to galaxy 2. Middle: Rotation curves for material originally belonging to galaxy 1 . Squares: total luminous matter from galaxy 1. Stars: material originally in the disk. Crosses: material originally in the bulge. Bottom: Rotation curves for material originally belonging to galaxy 1 . Triangles: total luminous matter from galaxy 2. Stars: material originally in the disk. Crosses: material originally in the bulge. The abscissa is in units of the luminous half-mass radius. For velocity units, see text. 


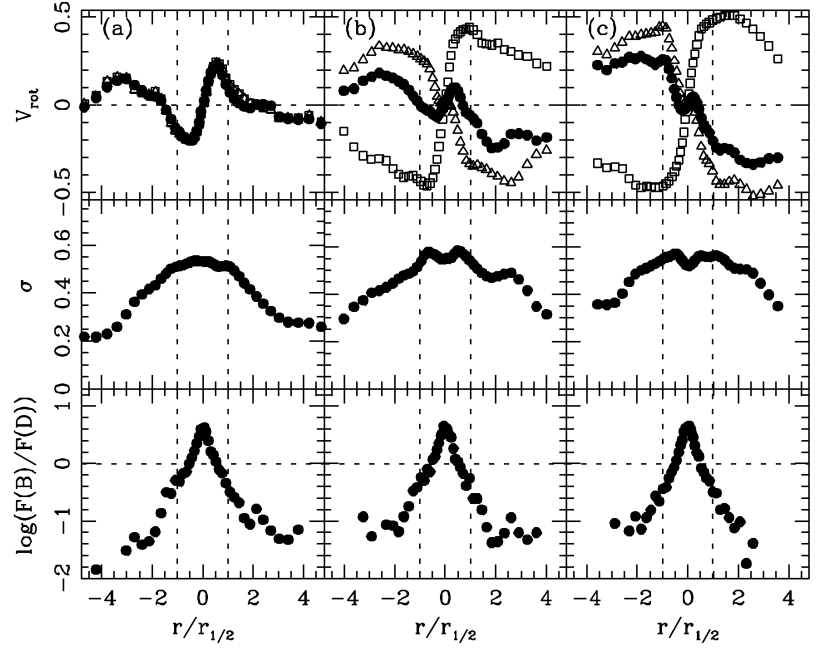

FIG. 2.-Kinematic properties for unequal mass mergers. (a) Model with $M_{2}: M_{1}=1$. (b) Model with $M_{2}: M_{1}=2$. (c) Model with $M_{2}: M_{1}=3$. Top: Rotation curves. Middle: Velocity dispersion profiles. Bottom: Surface density ratio of matter originally in the bulges to matter originally in the disks, along the slit. Symbols are as in Fig. 1.

ferred to the disk and halo material. The final rotation properties of particles initially belonging to the disks are set by the balance of spin and orbital angular momenta and the exchange with the bulges and halos. The orbital angular momentum [ $\boldsymbol{J}_{\text {orb }}$ $($ disk $)=0.87]$ dominates over the spin term $\left[J_{\text {spin }}(\right.$ disk $)=$ 0.26]. However, the merger orbit becomes increasingly radial at each pericenter passage as the dark halo absorbs $\boldsymbol{J}_{\text {orb }}$ (Barnes 1992). The result is a main body slowly rotating in the direction of the original disk spins. Only in the inner region $(0.50$ of the luminous half-mass radius) have velocities of disk material been reversed, a result of the fact that little disk and halo material is available to absorb the orbital angular momentum of the sinking bulges.

\section{SIZE OF THE KDC}

The radius $R_{\text {cr }}$ of the counterrotating region is basically set by the radius at which the material originally in the disks starts to dominate the remnant surface density. $R_{\mathrm{cr}}$ has little dependence on the orbital parameters and the initial spin orientation (see Table 3). In the canonical models with a bulge-to-disk ratio of 1:2, such as the one in Figure 1, $R_{\mathrm{cr}}$ is comparable to the luminous half-mass radius $R_{1 / 2}$ and hence larger than the effective radius $R_{e}$.

Although elliptical galaxies with $R_{\text {cr }}>R_{e}$ may exist (measured rotation curves rarely reach $R_{e}$ ), typical values for observed $R_{\text {cr }}$ 's are a few tenths of $R_{e}$ (e.g., Balcells 1992). Hence it is useful to investigate whether, by varying model parameters, $R_{\text {cr }}$ reaches the values below $R_{e}$ typical of observed systems.

We find that $R_{\text {cr }}$ becomes smaller for galaxy mass ratios somewhat different from unity. Masses cannot be made very different, otherwise the larger of the disks is not entirely destroyed and the merger outcome is not an elliptical galaxy. For mass ratios 1:2 and 1:3 (models 5 and 6), the counterrotating region becomes smaller than $R_{e}$ (Fig. 2; Table 3). This suggests that this mechanism is capable of producing KDCs with sizes comparable to those of real systems.

In the 1:3 mass merger, we find that the outer portions of the surface density profile have an exponential behavior. Ob-

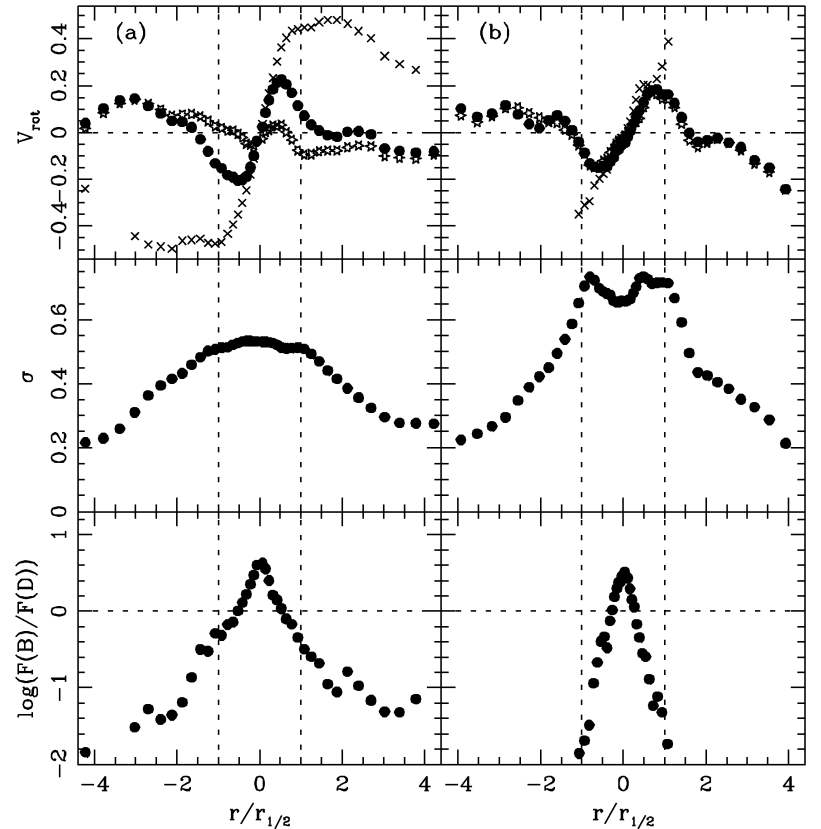

FIG. 3.-Kinematic properties for models with differing bulge masses. (a) Model with $M_{B} / M_{D}=0.5$. (b) Model with $M_{B} / M_{D}=0.2$. Top: Rotation curves. Middle: Velocity dispersion profiles. Bottom: Surface density ratio of matter originally in the bulges to matter originally in the disks, along the slit. Symbols are as in Fig. 1.

servationally, such an object would be classified as a bulgedominated S0 galaxy. Hence, retrograde, intermediate-mass mergers may provide a mechanism for the onset of nuclear counterrotation in S0 galaxies.

\section{SMALLER BULGES}

Given that the bulge material determines the counterrotation signature, we might suspect that galaxies with smaller bulgeto-disk ratios may result in a smaller counterrotating region. This turns out not to be the case. Figure 3 compares the rotation curve of merger 3 (left; $M_{B} / M_{D}=0.5$ ) to that of merger 8 (right; $M_{B} / M_{D}=0.2$ ). The behavior of the disk material is quite different in the two cases. In merger 3, the disk material behaves as described in § 3: except for a small central region of reversed rotation, its rotation is a relic of the initial spin of the disk. In merger 8 , the region of reversed rotation extends well beyond the half-mass radius $R_{1 / 2}$, and $R_{\mathrm{cr}}: R_{1 / 2}$ is similar in both models (see Table 3). In the small-bulge models, the weaker memory of the initial spin of the disks is due to the development of a large-scale nonaxisymmetric pattern in response to the tidal field (e.g., Hernquist 1992). This pattern removes spin from the disks, transports it outward, and $\boldsymbol{J}_{\text {orb }}$ ends up as the main contributor to the rotation curve. Hernquist (1993) noted that dense central bulges can stabilize the disk against tidally induced bar formation. This appears to occur in our massive bulge mergers (Fig. 3, left).

We cannot infer lower limits on $R_{\text {cr }}$ from these arguments because the fate of the angular momentum of the bulge and the disk during the merger depends not only on their masses but also on their densities, as well as on those of the halo. More centrally concentrated halos would contribute to the disk stability.

The central panels in Figures 2 and 3 show the velocity 
dispersion profiles $\sigma(r)$ in the models. The profiles are roughly flat within $R_{1 / 2}$. Differences in halo parameters caused by the differences in bulge mass are responsible for the higher central dispersion of the small-bulge model (Fig. 3, right). The bottom panels in Figures 2 and 3 show the ratio of bulge to disk surface densities along the slit. Outside of the counterrotating region, this ratio drops below 0.1 , making the extended counterrotating material undetectable by present-day line profile analysis techniques such as Gauss-Hermite fitting (van der Marel \& Franx 1993; Zhao \& Prada 1996) and unresolved Gaussian decomposition (Kuijken \& Merrifield 1993).

\section{DISCUSSION}

The model presented here provides a new mechanism to form KDCs. Like the model of HB91, the KDC forms in a major merger, but the physics is different: we invoke stellar dynamical processes, while the HB91 model relies on hydrodynamical evolution to form a nuclear gaseous disk. Both mechanisms may occur in nature. We may tentatively infer from the core sizes that the HB91 model applies to small KDCs and our model applies to large KDCs.

Our modest exploration of parameter space suggests that orbital and disk inclination parameters are not critical. Noteworthy is the small dependence on $\boldsymbol{J}_{\text {orb }}$, which indicates that the process does not rely on nearly radial orbits to preserve the spin orientation of the disk material. Trial runs suggest that adding rotation to the bulge models does not modify our results in a fundamental way, although the rotation curves tend to become more complex. Analysis of rotating bulge mergers is beyond the scope of this Letter.

Our model avoids the need to build the entire counterrotating component in the aftermath of a starburst. The KDC population is directly related to the precursors' stellar populations. We thus approach line-strength indicators of KDC elliptical galaxies in terms of those of bulges and disks. Color-derived ages of bulges and inner disks are similar (Peletier \& Balcells 1996), but line strengths and line ratios differ in bulges and disks (Fisher, Franx, \& Illingworth 1996); bulges show an inward rising Mg profile and over-solar $[\mathrm{Mg} / \mathrm{Fe}]$ ratio. Because the rapid decrease of bulge fraction with radius in the merger remnant, our model predicts the steep $\mathrm{Mg}$ profile and an over-solar $[\mathrm{Mg} / \mathrm{Fe}]$ core observed in KDC cores. Hence, the metallicity indicators of KDCs may be accounted for directly in terms of those of the precursor stellar populations. Quantitative estimates will be presented elsewhere. Matching the high metallicities of elliptical cores may be difficult, but we expect the gas component in the precursor spirals to undergo a starburst, which pumps up the remnant metallicity.

Our results suggest an explanation for counterrotating cores in lenticular galaxies in terms of mergers of unequal galaxies, since, for mass ratios above $3: 1$, the disk is not destroyed, but the counterrotating core forms nevertheless. If sufficient cool gas remains in the system to rebuild the disk (Kauffmann, Guiderdoni, \& White 1993), an early-type spiral with a counterrotating bulge may result. This mechanism could explain the counterrotating bulge in NGC 7331 (Prada et al. 1996).

Although stemming from the same physics, the present model is different from the eE model (BQ90) in several respects. Because in eE mergers a giant elliptical galaxy is already in place before, matching real elliptical galaxies afterward is easy, whereas the S-S merger takes longer to relax into a smooth light distribution. More significantly perhaps, eE mergers do not easily account for population properties of KDCs, whereas $\mathrm{S}-\mathrm{S}$ stellar mergers generate the color and metallicity profiles of elliptical galaxies quite naturally.

We thank Lars Hernquist for making his version of the tree code available to us and the anonymous referee for useful comments.

\section{REFERENCES}

Balcells, M. 1990, in Morphological and Physical Classification of Galaxies, ed. G. Longo, M. Capaccioli, \& G. Busarello (Dordrecht: Kluwer), 221

Balcells, M., \& Quinn, P. J. 1992, ApJ, 361, 381 (BQ90)

Barnes, J. H. 1992, ApJ, 393, 484

Davies, R. L., Sadlier, E. M., \& Peletier, R. F. 1993, MNRAS, 262, 650

Fisher, D., Franx, M., \& Illingworth, G. D. 1996, ApJ, 459, 110

Hau, G. K. T., Carter, D., \& Balcells, M. 1998, MNRAS, in press (HCB)

Hernquist, L. 1990, J. Comp. Phys., 87, 137

1992, ApJ, 400, 460

1993, ApJ, 409, 548
Hernquist, L., \& Barnes, J. E. 1991, Nature, 354, 210 (HB91)

Kauffman, G., Guiderdoni, B., \& White, S. D. M. 1993, MNRAS, 264, 201

Kuijken, K., \& Dubinski, J. 1995, MNRAS, 277, 1341

Kuijken, K., \& Merrifield, M. R. 1993, MNRAS, 262, 712

Peletier, R. F., \& Balcells, M. 1996, AJ, 111, 2238

Prada, F., Gutierrez, C. M., Peletier, R. F., \& McKeith, C. D. 1996, ApJ, 463, L9

Surma, P., \& Bender, R. 1995, A\&A, 298, 405

van der Marel, R. P., \& Franx, M. 1993, ApJ, 407, 525

Zhao, H.-S., \& Prada, F. 1996, MNRAS, 282, 1223 